Comparing plasma fluid models of different order for 1D streamer ionization fronts

This content has been downloaded from IOPscience. Please scroll down to see the full text.

2015 Plasma Sources Sci. Technol. 24065002

(http://iopscience.iop.org/0963-0252/24/6/065002)

View the table of contents for this issue, or go to the journal homepage for more

Download details:

IP Address: 131.155.2.68

This content was downloaded on 08/10/2015 at 19:31

Please note that terms and conditions apply. 


\title{
Comparing plasma fluid models of different order for 1D streamer ionization fronts
}

\author{
Aram H Markosyan ${ }^{1,2}$, Jannis Teunissen ${ }^{2}$, Saša Dujko ${ }^{2,3}$ and Ute Ebert ${ }^{2,4}$ \\ ${ }^{1}$ Electrical Engineering and Computer Science Department, University of Michigan, Ann Arbor, MI \\ 48109, USA \\ 2 Centrum Wiskunde and Informatica (CWI), PO Box 94079, 1090 GB Amsterdam, The Netherlands \\ 3 Institute of Physics, University of Belgrade, PO Box 68, 11080 Zemun, Belgrade, Serbia \\ ${ }^{4}$ Department of Applied Physics, Eindhoven University of Technology, PO Box 513, $5600 \mathrm{MB}$ \\ Eindhoven, The Netherlands \\ E-mail: armarkos@umich.edu
}

Received 23 March 2015, revised 6 August 2015

Accepted for publication 7 September 2015

Published 8 October 2015

\begin{abstract}
We evaluate the performance of three plasma fluid models: the first order reaction-drift-diffusion model based on the local field approximation; the second order reaction-drift-diffusion model based on the local energy approximation and a recently developed high order fluid model by Dujko et al (2013 J. Phys. D 46 475202) We first review the fluid models: we briefly discuss their derivation, their underlying assumptions and the type of transport data they require. Then we compare these models to a particle-in-cell/Monte Carlo (PIC/MC) code, using a 1D test problem. The tests are performed in neon and nitrogen at standard temperature and pressure, over a wide range of reduced electric fields. For the fluid models, transport data generated by a multi-term Boltzmann solver are used. We analyze the observed differences in the model predictions and address some of the practical aspects when using these plasma fluid models.
\end{abstract}

Keywords: streamer discharges, modeling, convection diffusion reaction, high order model, particle in cell, Monte Carlo

(Some figures may appear in colour only in the online journal)

\section{Introduction}

The development of models and simulation techniques for electrical discharges has been ongoing for more than five decades. Here we focus on models for streamer discharges, which are rapidly growing ionized channels that are non-linearly controlled by space charge effects. In these discharges, electric fields and electron energies are getting particularly high in the ionization front. Streamers occur in nature (lightning and sprites [2,3]) and technology (plasma-assisted combustion [4], plasma medicine [5], disinfection [6] etc). Streamer models can be categorized into four types: particle models, kinetic models, fluid models and hybrid models.

Particle models are typically of the particle-in-cell (PIC) type. With these models, a large number of particles is followed as they move through the simulation domain, so that one has direct information about the particle distribution in phase space $[7,8]$. Particle models have few underlying assumptions or approximations, and therefore give accurate predictions over a wide range of conditions, but they are computationally relatively expensive. In recent years, particle codes that use graphics processing units (GPUs) have shown promising speed-ups [9].

The second type of models are the so-called kinetic models, that couple the full Boltzmann equation with the Poisson equation. Such models are computationally very costly, because they require a numerical grid that covers the full phase space. However, advances in computing power and algorithms have made some of these fully kinetic simulations possible [10, 11].

The third type of models are the plasma fluid models, which describe the electron dynamics in plasma based on macroscopic quantities like electron density, average electron velocity, average electron energy etc. Fluid models 
are typically constructed by taking the velocity moments of the Boltzmann equation. Depending on the number of moments considered and the closure assumptions, different fluid models have been derived over the last four decades $[1,12-16]$. Fluid models require less computational resources than particle models, yet they can provide reasonably accurate results.

Finally, hybrid models combine the strengths of the fluid and particle approaches, by combining the fast speed of fluid simulations with the accurate particle kinetics of particle models. Different types of hybrid models can be constructed. In some applications, a particle model is used to follow high-energy electrons while low energy electrons and ions are treated as fluid [17]. Alternatively, one can use different models in different spatial regions [18].

In the present paper we consider three plasma fluid models: the first order reaction-drift-diffusion model based on the local field approximation; the second order reaction-drift-diffusion model based on the local energy approximation and a recently developed high order fluid model. We investigate how well these models can simulate ionization waves in 1D, by comparing them with a PIC code. Such ionization waves can be seen as the $1 \mathrm{D}$ version of streamer channels.

In [19], part of this model comparison has already been carried out. Here, we extend our previous work in the following way:

- Compared to [19], we now include an additional model, namely the second order reaction-drift-diffusion model with an energy equation.

- In [19], simulations were carried out in nitrogen only, here we consider both neon and nitrogen at standard temperature and pressure (STP). The major difference between these two gases is that neon is an atomic gas and nitrogen a molecular gas, see also the discussion below.

- Here, we also discuss some of the more practical aspects that are important when using the models.

Compared to nitrogen, neon has fewer inelastic channels, with higher threshold energies. Because neon is an atomic gas, it does not have the vibrational and rotational excitations of nitrogen. This means that, even though the ionization energy is higher, neon discharges can be generated in lower electric fields, due to the smaller energy loss in inelastic channels. Furthermore, certain non-local phenomena, such as a high electron energy in the streamer channel (despite the electrical screening there), are more pronounced. Neon is also frequently used in industry (e.g. neon lamps, plasma medicine).

In this paper we use a one-dimensional ionization wave as a test problem. An ionization wave forms when an electric field above the breakdown threshold is applied. Electrons move in the field and produce electron-impact ionization, and after some time the degree of ionization is sufficient to create an electrically screened region. As mentioned above, such ionization waves can be seen as the one-dimensional equivalent of streamer channels [20]. There are a couple of important differences between one-dimensional ionization waves and streamers, however:
- Streamer channels enhance the electric field at their tips, just like elongated conductors. This field enhancement depends on the curvature of the streamer head, and is therefore missing in a one-dimensional ionization wave without curvature.

- The electric field ahead of an ionization wave does not naturally decay like it does for a streamer with local field enhancement. This can make the 1D ionization wave a harder test problem than 'streamer' tests in 2D/3D: the growth in the whole region ahead of the front needs to be accurately described [21].

- As a streamer channel grows, more charge is required at its tips to screen the interior electric field. This generates an internal current, and therefore an internal electric field. For an ionization wave in a constant background field no such current is required, and its internal field is completely screened.

The structure of the paper is as follows. In section 2, we discuss the origin of the different fluid models (the Boltzmann equation). In sections 2.1 and 2.3, we briefly summarize the derivation and the underlying assumptions of the first and high order fluid model. In section 2.2, we present the second order fluid model. We shortly discuss the particle model in section 5. In section 5 the transport data for neon, which are used in the fluid models, are described in some detail. The simulation conditions that we use for comparing the models are discussed in section 5, and the results of the comparison are given in section 6 . In section 7 we discuss our results in more detail and we give our conclusions in section 8 .

\section{Fluid models for streamer discharges}

The dynamics of a system of charged particles can be described microscopically by the Boltzmann equation or by the Particle-in-Cell Monte Carlo (PIC/MC) technique. While the latter one follows individual particles in phase space [22], the former one describes the ensemble of particles by the distribution function, or phase density, $f_{i}(\boldsymbol{r}, \boldsymbol{c}, t)$ in phase space $(\boldsymbol{r}, \boldsymbol{c})$ for particle species $i$ at time $t$. The evolution of distribution function is described by the Boltzmann equation [23-26]:

$$
\partial_{t} f_{i}+\boldsymbol{c} \cdot \nabla f_{i}+\frac{e_{i}}{m_{i}} \boldsymbol{E} \cdot \nabla_{c} f_{i}=-J\left(f_{i}, f_{0}\right) .
$$

where $\nabla$ is the differential operator with respect to space $r$ and $\nabla_{\boldsymbol{c}}$ with respect to velocity $\boldsymbol{c}, e_{i}$ and $m_{i}$ are charge and mass of species $i$, and $t$ is time. The right-hand side of equation (1), $J\left(f_{i}, f_{0}\right)$, describes the collisions of charged particles with neutral molecules, accounting for elastic, inelastic, and non-conservative (e.g. ionizing or attaching) collisions, and $f_{0}$ is the velocity distribution function of the neutral gas (usually taken to be Maxwellian at fixed temperature).

If we work under electrostatic conditions, then space charge effects can be accounted for by coupling the Boltzmann equation to Poisson's equation:

$$
\nabla^{2} V=-\frac{1}{\epsilon_{0}} \sum_{i} q_{i} n_{i}(\boldsymbol{r}, t),
$$


so that the electric field can be calculated by:

$$
\boldsymbol{E}=-\nabla V
$$

where $V$ is electric potential, $\epsilon_{0}$ is the dielectric permittivity, and $q_{i}$ and $n_{i}$ are the charge and density of species $i$ (electrons, ions, excited states etc). One should note that the Boltzmann equation provides a complete description of the distribution in phase space of species $i$, while the density $n_{i}$ in equation (2) is a macroscopic (averaged) quantity. The number density $n_{i}$ can be directly calculated from the distribution function $f_{i}(\boldsymbol{r}, \boldsymbol{c}, t)$ :

$$
n_{i}(\boldsymbol{r}, t)=\int f_{i}(\boldsymbol{r}, \boldsymbol{c}, t) \mathrm{d} \boldsymbol{c} .
$$

For most applications, especially those that require two or three spatial dimensions, it is not feasible to solve the system (1)-(3) with direct numerical simulations. An alternative approach is to consider the velocity moments of distribution function:

$$
\langle\phi(\boldsymbol{c})\rangle=\frac{1}{n(\boldsymbol{r}, t)} \int \phi(\boldsymbol{c}) f(\boldsymbol{r}, \boldsymbol{c}, t) \mathrm{d} \boldsymbol{c}
$$

with $\phi(\boldsymbol{c})=1, m \boldsymbol{c}, \frac{1}{2} m c^{2}, \frac{1}{2} m c^{2} \boldsymbol{c}, \ldots$ giving the average velocity $\boldsymbol{v}=\langle\boldsymbol{c}\rangle$, average energy $\varepsilon=\left\langle\frac{1}{2} m c^{2}\right\rangle$, average electron energy flux $\boldsymbol{\xi}=\left\langle\frac{1}{2} m c^{2} \boldsymbol{c}\right\rangle$ and so on. \langle\rangle represents the average over the velocity $\boldsymbol{c}$ of the charged particles. Using this approach, the set of moment equations can be found by multiplying (1) by $\phi(\boldsymbol{c})$ and integrating over the velocity space:

$$
\partial_{t}(n\langle\phi(\boldsymbol{c})\rangle)+\nabla \cdot(n\langle\boldsymbol{c} \phi(\boldsymbol{c})\rangle)-n \frac{e}{m} \boldsymbol{E} \cdot\left\langle\nabla_{\boldsymbol{c}} \phi(\boldsymbol{c})\right\rangle=C_{\phi},
$$

where $C_{\phi}$ is the collision term:

$$
C_{\phi}=-\int \phi(c) J(f) \mathrm{d} c .
$$

To derive the term $\left\langle\nabla_{\boldsymbol{c}} \phi(\boldsymbol{c})\right\rangle$, a partial integration over $\boldsymbol{c}$ has been performed.

In the following subsections we present different models with respect to the number of moments considered. Except for the high order model, these models have been frequently used over the last few decades. We give a short list with the main assumptions present in the models.

\subsection{The first-order or classical model}

The first order model, also called classical model, is the simplest and most used model considered in this work [1, 15, 17, 20, 27-33]. For the full and strict derivation we refer to [1, 15]. This model considers only the first two balance laws from the system (6). For electrons and ions it reads as:

$$
\begin{gathered}
\partial_{t} n=\nabla \cdot(\mu(E) n \boldsymbol{E}+D(E) \cdot \nabla n)+n \nu_{I}(E), \\
\partial_{t} n_{\text {ion }}=n \nu_{I}(E),
\end{gathered}
$$

where $E=|\boldsymbol{E}|, n_{\text {ion }}$ is ion density and where mobility $\mu$, diffusion $D$ and $\nu_{I}$ are functions of the local electric field. In equation (9) we assume that the displacement of ions is negligible (e.g. when considering short time scales). Of course, the system (8) and (9) is coupled to the Poisson equation (2) and(3) which in 1D has the following simple form:

$$
\frac{\partial E}{\partial x}=\frac{e}{\epsilon_{0}}\left(n_{\text {ion }}-n\right) .
$$

During derivation of this model the following assumptions are made:

(i) the distribution of random velocities is close to isotropic. This is a strong assumption for streamer discharges, where at the streamer tip the electric field is locally very high and strong pressure gradients exists,

(ii) the momentum transfer by collisions $C_{m \mathbf{c}}=-n m \nu_{\mathrm{eff}} \boldsymbol{v}$, where $\nu_{\text {eff }}$ is the effective momentum transfer collision frequency, which accounts for momentum transfer exchange only in elastic and inelastic collisions,

(iii) the source term in the mass balance equation is $C_{1}=n \nu_{I}$, where $\nu_{I}$ is the ionization collision frequencies due to electron-molecule collisions, for non-attaching gases.

(iv) the rate of momentum change is smaller than the rate of momentum transfer $\nu_{\text {eff }}$,

(v) as a further simplification all transport properties are functions of the local electric field, i.e. the Local Field Approximation (LFA) is used.

(vi) the system is close to equilibrium and the NernstTownsend-Einstein relation $D / \mu=k T / e$ is valid, where $D$ is the diffusion constant, $\mu$ is the electron mobility and $T$ is the electron temperature,

We would like to remark that, when the classical fluid model is derived from the Boltzmann equation (1), the diffusion coefficient, strictly speaking, is a scalar. But in the case of streamer discharges, the diffusion tensor can be strongly anisotropic. A few authors have considered this anisotropic diffusion [18, 34]. Particularly, in [34] the effect of anisotropic diffusion on branching phenomena of negative streamers is investigated. In $[18,35,36]$ a phenomenological extension of the classical fluid model based on a gradient expansion of electron density is provided. In what follows, the classical fluid model will be denoted by LFA.

\subsection{The second-order model}

The second order model also considers the energy balance equation. Depending on the closure assumption, one can obtain conceptually different second-order models. In this section we discuss the model called LEA (Local Energy Approximation), which originates (to our knowledge) from [37-39]. This model has been used by many others in different applications $[13,17,18,40-50]$ and reads as follows:

$$
\begin{gathered}
\partial_{t} n=-\nabla \cdot \boldsymbol{\Gamma}+n \nu_{I}(\varepsilon), \\
\partial_{t} n_{\text {ion }}=n \nu_{I}(\varepsilon), \\
\partial_{t}(n \varepsilon)=-\frac{5}{3} \nabla \cdot(\varepsilon \boldsymbol{\Gamma})+\boldsymbol{E} \cdot \boldsymbol{\Gamma}-n \sum_{j} k_{j} \varepsilon_{j},
\end{gathered}
$$




$$
\boldsymbol{\Gamma}=-n \mu(\varepsilon) \boldsymbol{E}-D(\varepsilon) \nabla n .
$$

The following assumption underline in derivation of this model:

(i) the assumptions (i)-(vi) from the section 2.1 are valid, i.e we have equation (8), but with transport parameters depending on local average electron energy,

(ii) the total energy transfer (losses) is due to the elastic and inelastic collisions:

$$
C_{\varepsilon}=n \sum_{j} k_{j} \varepsilon_{j},
$$

where $k_{j}$ and $\varepsilon_{j}$ are the collision rate coefficient and energy loss per electron per collision (elastic, inelastic) for the collision process denoted by $j$,

(iii) the pressure tensor is simplified to $\boldsymbol{P}=n k \boldsymbol{T} \approx \frac{2}{3} n \varepsilon \boldsymbol{I}$,

(iv) the heat flux vector $\boldsymbol{q}$ is assumed to be proportional to the gradient of the electron average energy $\boldsymbol{q}=-\frac{5}{3} n D \nabla \varepsilon$, where $D$ is the diffusion coefficient,

(v) all transport properties are functions of the local average electron energy, i.e. the Local Energy Approximation (LEA) is used.

This system is again coupled with the Poisson equation (10). As illustrated by Hagelaar and Pitchford [40], the LEA model is consistent with classical theory for electron transport based on the two term approximation for solving the Boltzmann equation.

Markosyan et al [19] have investigated the importance of the energy flux equation by deriving a second-order model with neglected energy flux term. A similar system has been derived by Kanzari et al [14]. Eichwald et al [13] used a similar approach to simulate streamer dynamics and radical formation in a pulsed corona discharge used for flue gases. Guo and $\mathrm{Wu}$ [49] have developed a more sophisticated second order model in which the Langevin theory was used to simplify the collision source terms with a priori knowledge of the relaxation times of electron energy and momentum. Another, recent, second order model has been derived by Becker et al [51] as a simplification of their more complete model containing four moments [16]. They illustrate that this simplification can be done without loss of accuracy, if the characteristic frequency of the electric field alteration in the discharge is small in comparison with the momentum dissipation frequency of the electrons [16].

\subsection{The high-order model}

The high-order fluid model considered in this paper has recently been derived in $[1,19]$, where the infinite system of moment equation (6) is truncated at the level of energy flux balance by approximating the pressure tensor with a scalar kinetic pressure. The collisional terms were evaluated using momentum transfer theory [52-54]. For more details of the derivation we refer to [1]. The high order fluid model consists of balance laws for the electron density $n$, for the average electron velocity $\boldsymbol{v}$, for the average electron energy $\varepsilon$ and for the average electron energy flux $\boldsymbol{\xi}$ :

$$
\begin{gathered}
\frac{\partial n}{\partial t}+\nabla \cdot n \boldsymbol{v}=n \nu_{I}, \\
\frac{\partial}{\partial t}(n \boldsymbol{v})+\frac{2}{3 m} \nabla(n \varepsilon)-n \frac{e}{m} \boldsymbol{E}=-n \boldsymbol{v}\left(\nu_{m}+\nu_{I}\right), \\
\frac{\partial}{\partial t}(n \varepsilon)+\nabla \cdot(n \boldsymbol{\xi})-e \boldsymbol{E} \cdot(n \boldsymbol{v})=-n \nu_{e}\left[\left(\varepsilon-\frac{3}{2} k T_{0}\right)+\Omega\right], \\
\frac{\partial}{\partial t}(n \boldsymbol{\xi})+\nabla\left(\beta \frac{2 n}{3 m} \varepsilon^{2}\right)-\frac{5}{3} n \varepsilon e \boldsymbol{E}=-\nu_{m} n \boldsymbol{\xi}
\end{gathered}
$$

where $\boldsymbol{E}$ is the electric field, $m$ and $e$ are electron mass and charge, $T_{0}$ is gas temperature and $k$ is the Boltzmann constant. The average collision frequencies for momentum $\nu_{m}$ and energy transfer in elastic collisions $\nu_{e}$ are defined in [1], $\nu_{I}$ is the ionization rate coefficient. The term $\Omega$ represents the average energy lost in one energy relaxation time $\nu_{e}^{-1}$ and is given in [1]. $\beta$ is a parameter introduced to approximate the high order tensors in the energy flux equation in terms of lower moments [1].

In the system (16)-(19) the following assumptions are present:

(i) $C_{1}=n \nu_{I}$,

(ii) the momentum transfer approximation is employed [1] to evaluate collisional terms,

(iii) the pressure tensor is simplified to $\boldsymbol{P}=n k \boldsymbol{T} \approx \frac{2}{3} n \varepsilon \boldsymbol{I}$,

(iv) the temperature tensor is isotropic, and hence $\langle\boldsymbol{c c}\rangle \approx \frac{\left\langle c^{2}\right\rangle}{3} \boldsymbol{I}$

(v) the higher order tensor $\left\langle c^{2} c \boldsymbol{c}\right\rangle$ appearing in the energy flux balance equation can be expressed by a product of the lower order moments as $\left\langle c^{2} \boldsymbol{c c}\right\rangle \approx \beta\left\langle c^{2}\right\rangle\langle\boldsymbol{c c}\rangle \approx$ $\beta\left\langle c^{2}\right\rangle \frac{\left\langle c^{2}\right\rangle}{3} \boldsymbol{I}=\beta \frac{4}{3 m^{2}} \varepsilon^{2} \boldsymbol{I}$. $\beta$ is a parametrization factor, generically close to unity, when the higher order correlation term $\left\langle c^{2} \boldsymbol{c c}\right\rangle-\left\langle c^{2}\right\rangle\langle\boldsymbol{c c}\rangle$ can be neglected [1, 19],

(vi) following original papers $[1,19]$, in this work $\beta$ is considered to be equal to 1 .

As we have already mentioned, an alternative approach has been described by Becker et al [16].

\section{The MC particle model}

As a reference model we use a Particle-in-Cell Monte Carlo (PIC/MC) code. We assume that PIC/MC model can simulate the full physics of ionization waves in $1 \mathrm{D}$, so that agreement between a fluid model and the PIC/MC results can validate the fluid model.

Of course, the cross-sections used in the PIC code also have to be used to generate the coefficients of the fluid model (e.g. the mobility or ionization rate). This is discussed in more detail in the next section.

The construction of a planar front is straightforward in fluid models, as the spatial derivatives are simply evaluated in one direction only. However, in the particle model electrons move in all three spatial dimensions and hence, the 
three-dimensional setting has to be restricted as in previous work [7, 19].

\section{Transport parameters}

In this section we briefly discuss how the transport data for electrons are calculated and implemented in our fluid models of negative planar streamer fronts. The electron transport data employed in this work are calculated using a multi-term theory for solving the Boltzmann equation. The methods and techniques are by now standard and the readers are referred to our previous work $[23,52]$.

Transport and reaction data for electrons in $\mathrm{N}_{2}$ have been recently published $[1,55,56]$ and in this paper the emphasis is placed upon the data for electrons in Ne. Calculations of transport data at a temperature of $293 \mathrm{~K}$ are performed for reduced electric fields $\left(E / n_{0}\right)$ ranging from $1 \times 10^{-4}-1 \times 10^{3}$ $\mathrm{Td}\left(1 \mathrm{Td}=1 \times 10^{-21} \mathrm{Vm}^{2}\right)$. The first-order model is based on the local field approximation; it requires the electron mobility, diffusion coefficient and ionization rate as a function of the reduced electric field $E / n_{0}$ (where $n_{0}$ is the gas number density). The second-order model is based on the local mean energy approximation and the transport data, including electron mobility, diffusion coefficient and ionization rate as well as rate coefficients for relevant processes are functions of the electron mean energy. The correspondence between the mean energy and $E / n_{0}$ is used to find transport data for a given mean energy. The high-order fluid model requires average collision frequencies for momentum and energy transfer in elastic and inelastic collisions, and rate coefficients for all collision processes as a function of the mean electron energy. As for the second-order model, the correspondence between the mean energy and $E / n_{0}$ is used to find the collision frequencies. The momentum transfer collision frequency has not been determined directly from the cross sections but rather from the following equation:

$$
\nu_{m}=\frac{e}{m \mu(\varepsilon)},
$$

where $e$ and $m$ are the electron charge and mass, respectively, and $\mu(\varepsilon)$ is the electron mobility which is here a function of the mean energy. Since momentum transfer theory $[1,52]$ is used to determine the transfer of energy in the fluid equations, we use the following expression for the average energy lost in one energy relaxation time $\nu_{e}^{-1}$, through non-elastic processes $[1,52]$

$$
\Omega(\varepsilon)=\frac{m_{0}}{m+m_{0}} \sum_{\alpha} \frac{\left(\nu_{\alpha}-\nu_{\alpha}^{(s)}\right)}{\nu_{e}} \epsilon_{\alpha}+\sum_{i} \frac{\nu_{I}^{(i)}}{\nu_{e}} \Delta \epsilon_{I}^{(i)} .
$$

The inelastic channels $\alpha$ are governed by threshold energies $\epsilon_{\alpha}$ and collision frequencies for inelastic and superelastic processes $\nu_{\alpha}$ and $\nu_{\alpha}^{(s)}$, respectively. All collision frequencies (including the ionization collision frequencies $\nu_{I}^{(i)}$ ) depend on the electron mean energy. The collision frequency for energy transfer in elastic collisions $\nu_{e}$ is defined by equation (43) in Dujko et al [1].

The PIC/MC model requires a set of cross sections for electron scattering in Ne. This work considers negative planar streamer fronts in Ne using the cross section set of [57], which includes seven cross sections for electronic excitations and cross section for ionization as well as cross section for momentum transfer in elastic collisions. Transport data required as input in the fluid models are calculated using the same set of cross sections for electron scattering in Ne.

In figure 1(a) we show the electron mobility (multiplied by the gas number density) as a function of the reduced electric field $E / n_{0}$, and in figure 1(b) the diffusion coefficient is shown. Variation of the ionization rate coefficient and mean energy are shown on panels, 1(c) and 1(d), respectively. We see that the ionization rate becomes significant at the higher values of $E / n_{0}$ when sufficient electrons have enough energy to undergo ionization. From the profiles of the mean energy, we observe four distinct regions of transport as $E / n_{0}$ increases. First, there is an initial plateau region where the electron energy is thermal $(3 k T / 2 \approx 0.038 \mathrm{eV})$. Second, there is a region of sharp rise as the electrons start to rapidly gain the energy from the electric field. Third, there is a second small plateau region due to large energy loss of the electrons as the inelastic channels become important. Finally, there is another region of rapid rise, as both the elastic and inelastic processes drop off with high energy, and the electrons start to rapidly gain energy from the strong electric field.

In figures 2(a) and (b) we display the rate coefficients for momentum transfer in elastic collisions and the average energy loss in one energy relaxation time $\nu_{e}^{-1}$, through nonelastic processes, respectively.

In figure 3(a) we show the rate coefficients as a function of the mean electron energy for all collision processes. We compare the rate coefficients for momentum transfer in elastic collisions, the rate coefficient for total inelastic rate (sum of all rates for inelastic processes without ionization) and the ionization rate in figure 3(b). The ionization rate is significant for relatively high mean energies (i.e. high $E / n_{0}$ ) and is essential for modeling of streamers. From figure 3(b) we see that the ionization rate dominates the total inelastic rate for mean energies higher than $25 \mathrm{eV}$ which reflects the energy dependence and magnitude of the cross sections for ionization and electronic excitation.

\section{Simulation conditions}

In this work we consider 1D geometry and we simulate negative planar fronts. All fluid models are simulated in noble gas $\mathrm{Ne}$ and molecular gas $\mathrm{N}_{2}$ at standard temperature and pressure (STP). The electric field $\boldsymbol{E}=E \hat{\boldsymbol{e}}_{x}$ (where $\hat{\boldsymbol{e}}_{x}$ is the unit vector in the $x$ direction) drives the dynamics. We take $E$ as a positive value; therefore electrons drift to the left, and negative streamer ionization fronts move to the left as well.

\subsection{Boundary conditions}

To create steady propagation conditions for the negative front, the electric field on the left boundary $x=0$ is fixed to the time independent value $E_{0}$ : 

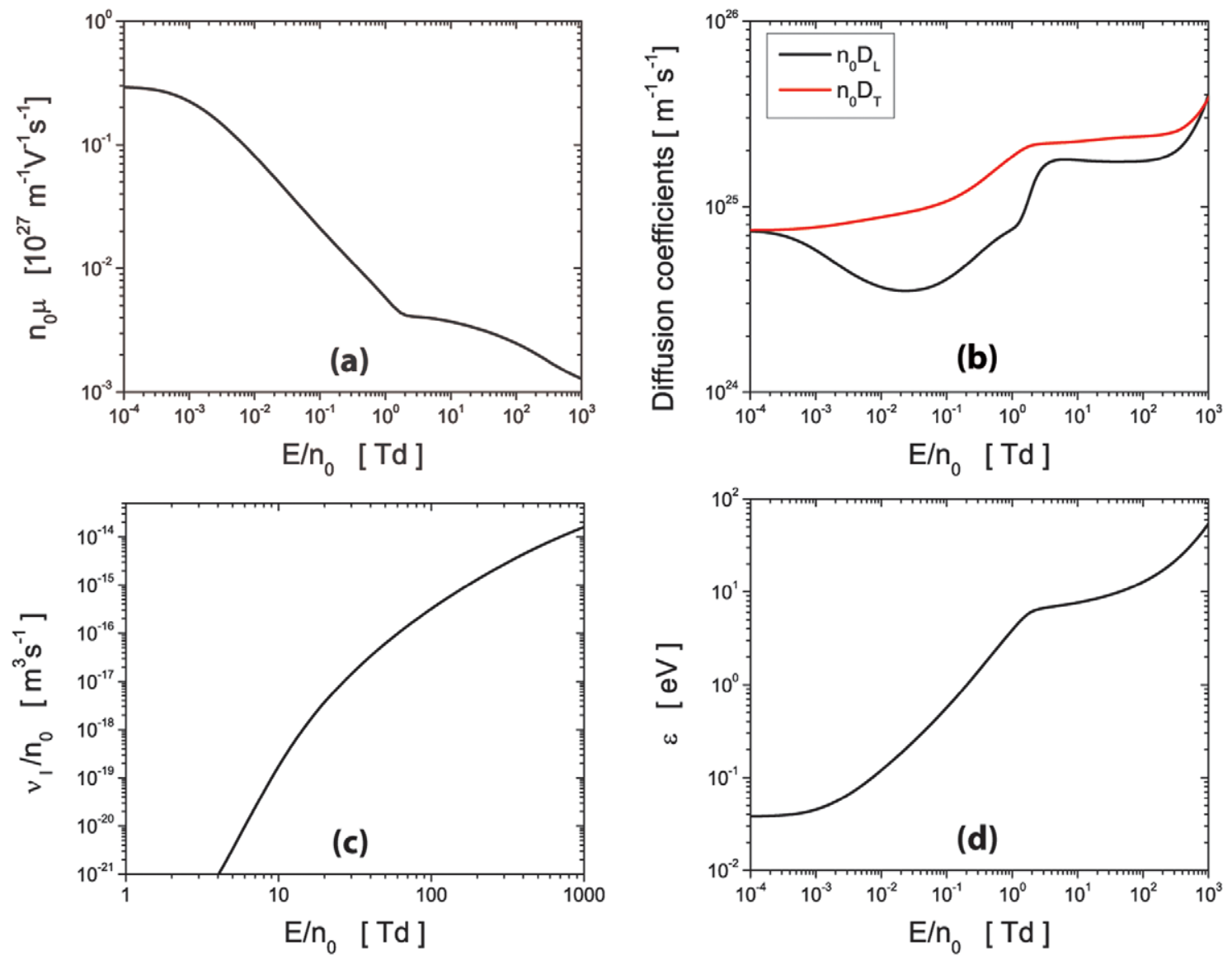

Figure 1. (a) Mobility, (b) longitudinal and transverse diffusion coefficient, (c) ionization rate and (d) mean energy, for electrons in Ne as a function of the reduced electric field $E / n_{0}$. These coefficients are used as input for the first-order model. The data are obtained from our multi-term solution of the Boltzmann equation.
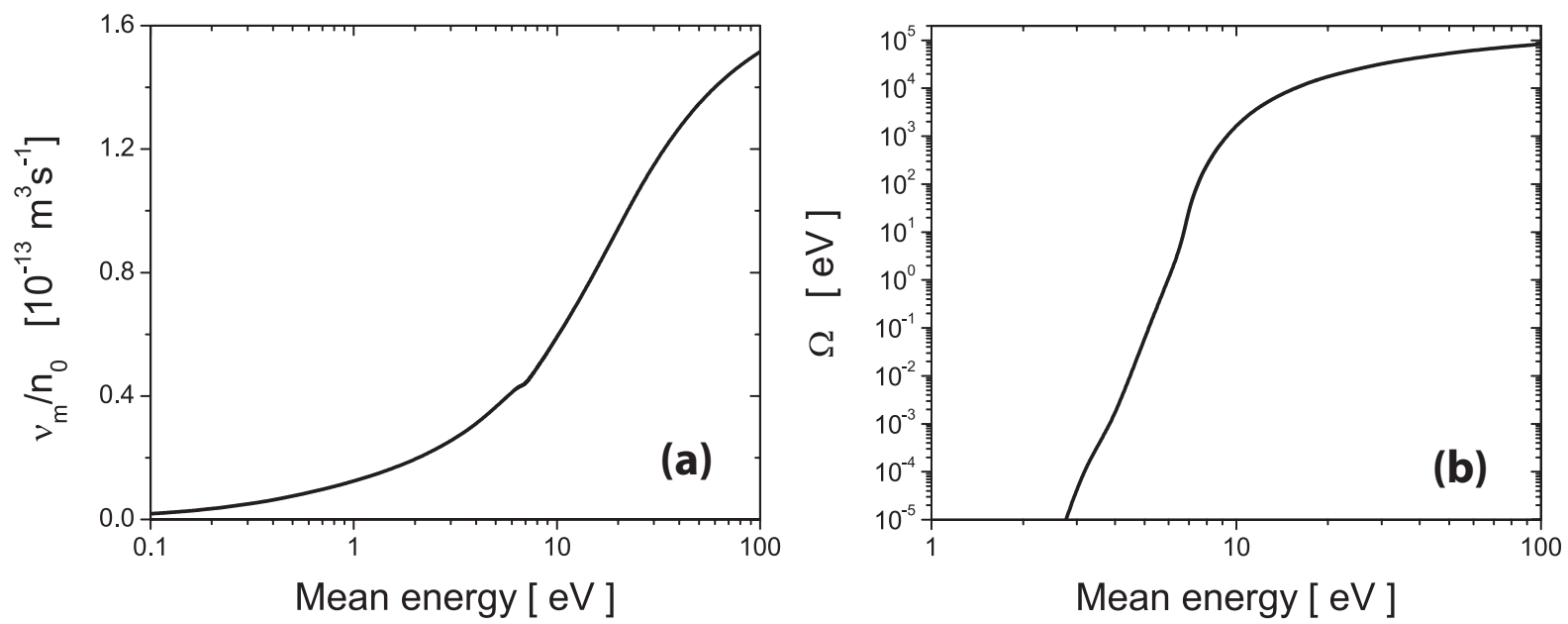

Figure 2. Input data for the high-order fluid model in neon: (a) Average collision frequency for momentum transfer in elastic collisions $\nu_{m}$, normalized by the neutral gas density $n_{0}$ and (b) the average energy lost in one energy relaxation time $\nu_{e}^{-1}$, through non-elastic processes. Both quantities are shown as a function of the mean electron energy.

$$
E(0, t)=E_{0}>0
$$

The electric field for $x>0$ is calculated by integrating equation (10) numerically over $x$, with (22) as a boundary condition. The right boundary is located at $x=L$; in all calculations we set the system length $L$ to $10 \mathrm{~mm}$ and we use 8500 grid points, so that the grid spacing is about $1.18 \mu \mathrm{m}$.

Homogeneous Neumann numerical boundary conditions are imposed for all conserved variables in all models on both of the ends of the system. However, all calculations end before 

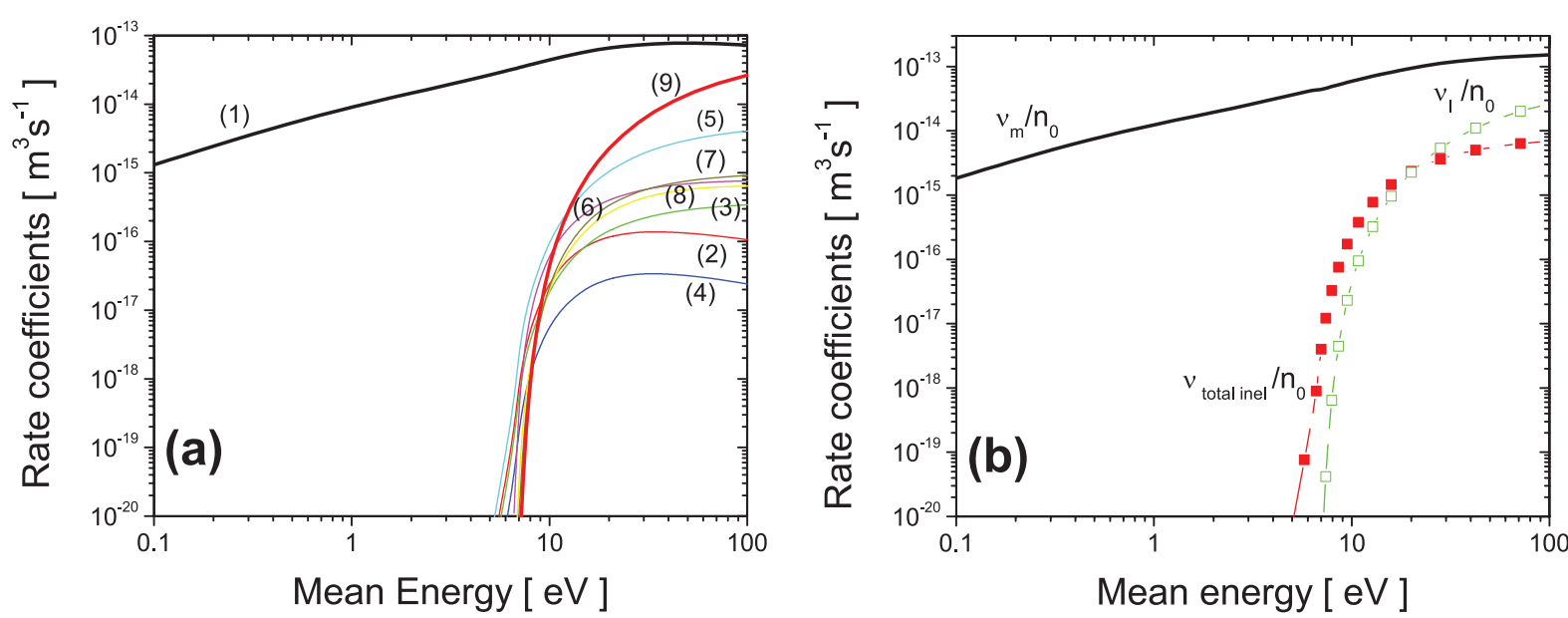

Figure 3. Rate coefficients (1-momentum transfer in elastic collisions, 2-8 electronic excitations and 9-ionization) in neon as a function of the electron energy, calculated with the multi-term solution of the Boltzmann equation and (b) momentum transfer rate, total inelastic rate and ionization rate. For reference, the ionization energy of neon is about $21.6 \mathrm{eV}$.

the ionization reaches the boundary, in other words, before the boundary conditions start to become relevant [58].

\subsection{Initial conditions}

We start all simulations with the same initial Gaussian distribution for electrons and ions

$$
\left.n(x)\right|_{t=0}=n^{*} \exp \left[-\frac{\left(x-x_{0}\right)^{2}}{\sigma^{2}}\right],
$$

where we have chosen $n^{*}=2 \times 10^{18} \mathrm{~m}^{-3}, x_{0}=9 \mathrm{~mm}$ and $\sigma=2.94 \times 10^{-5} \mathrm{~mm}$. The initial conditions for the average electron velocity, average electron energy and average electron energy flux are taken to be spatially homogeneous. These quantities are assumed to be relaxed to the background electric field when the simulation starts, with the values for the field given by a multi term solution of Boltzmann's equation, as already discussed in section 5 .

\subsection{Numerical method}

The LEA and LFA fluid models are spatially discretized using the scheme described in [59]. The high-order fluid model is discretized using FORCE scheme [60, 61]. As we already mentioned above, for all cases our spatial grid consisted of 8500 points, with a spacing of about $1.18 \mu \mathrm{m}$. Numerous studies have been devoted to the comparison of the accuracy of numerical schemes for advection problems in different situations (see e.g. [62-66]). In the present paper, we explicitly do not investigate the effect of the numerical schemes on the simulation models. Instead, we use a small enough time step and spatial resolution so that the results are essentially independent of the numerical scheme, see also section 7 .

\subsection{Time stepping}

For the time integration we use explicit trapezoidal rule for the LFA and LEA models, and the classical fourth-order RungeKutta 4 (RK4) scheme [67] for the high order model. With such an explicit time stepping method, there are typically at least three restrictions on $\Delta t$ [67-69]:

$$
\Delta t<C_{a} \Delta x / v \text { CFL condition, }
$$

$$
\Delta t<C_{d} \Delta x^{2} / D_{e} \text { explicit diffusion limit, }
$$

$$
\Delta t<\varepsilon /\left(\mu_{e} n_{e} e\right) \text { dielectric relaxation limit, }
$$

where $\Delta x$ is the spatial step size, $D_{e}$ the diffusion coefficient, $\varepsilon$ the permittivity, $\mu_{e}$ the electron mobility, $n_{e}$ the electron density and $e$ the elementary charge. $C_{a}$ and $C_{d}$ are the maximal Courant numbers for advection and diffusion equations [70]. The Courant number depends on the particular time-integration method and space discretization. Note that the first two conditions should actually be combined and that the last condition does not depend on the transport scheme used for the electrons.

The CFL time step restriction for the high order fluid model is given by the following formula

$$
\Delta t \leqslant C_{h} \frac{2 \Delta x}{\beta} \sqrt{\frac{3 m}{2 \max \varepsilon}},
$$

where $C_{h}$ is the maximal Courant number [19].

As stated above, we here focus on the accuracy of the models, not on their computational efficiency. Therefore, we have simply used a very small constant time step of $0.1 \mathrm{ps}$ for all models, satisfying all the above conditions.

\subsection{What to do when $n_{e} \rightarrow 0$ ?}

In many plasma fluid models the transport coefficients depend on the mean energy. However, the models typically contain equations for the evolution of the energy density $(Q=n \varepsilon)$. To get the mean energy at every point, we would like to simply compute

$$
\varepsilon=Q / n .
$$

Unfortunately, this simple expression leads to problems where $n \rightarrow 0$, not only because one can not divide by zero numerically, 

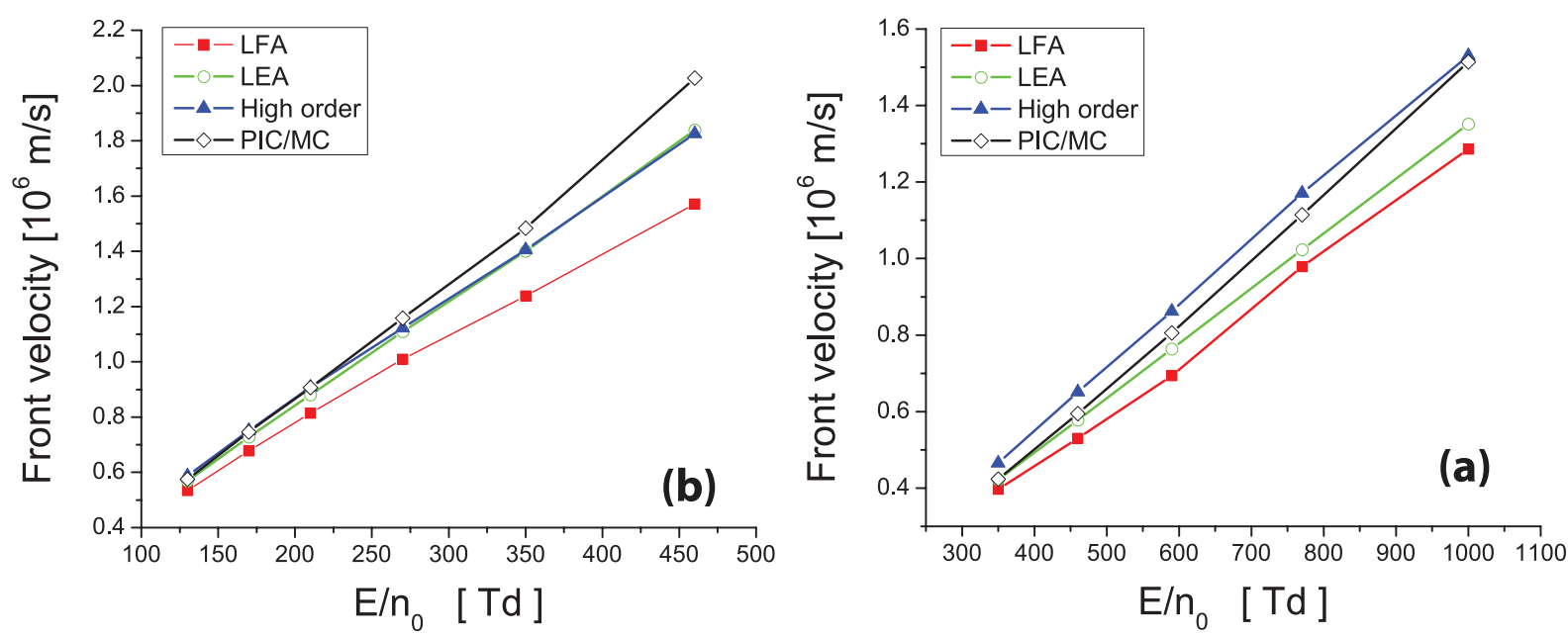

Figure 4. Velocities of planar fronts as a function of the electric field obtained with all the models in $\mathrm{Ne}$ (a) and in $\mathrm{N}_{2}$ (b). We remark that the negative sign of all velocities is removed here.

but also because round-off errors get large. Therefore, we use the following regularization:

$$
\varepsilon=\frac{Q+\eta_{0} \varepsilon_{f}(E)}{n+\eta_{0}},
$$

where $\varepsilon_{f}(E)$ is the relaxed mean energy in an electric field of strength $E$, and $\eta_{0}$ is a small density. Using equation (29) instead of equation (28) makes no difference where $n \gg \eta_{0}$. But where $n \lesssim \eta_{0}$, using (29) ensures that the mean energy is approximately relaxed to the background electric field. For the results shown in section 6 , we have used $\eta_{0}=10^{12} \mathrm{~m}^{-3}$.

\subsection{Implementation difficulties}

We here make use of explicit time stepping. There are two reasons for that: implicit schemes are tricky to implement for models that depend on tabulated input data and implicit schemes are computationally much more costly (per time step). For time-dependent simulations one is bound to CFLlike time step restrictions for accuracy, even with an implicit scheme. Therefore, explicit schemes are often more practical. On the other hand, the use of explicit time stepping has some implications for the numerical stability of the different models.

With the LFA model we did not encounter any problems. With the LEA model, oscillations in the electron energy occurred for the higher fields in nitrogen (see section 6). Such oscillations were also observed in [71], where the LEA model was described in quite some detail. Instead of using an implicit scheme, as in [71], we have increased the value of $\eta_{0}$ and decreased the time step, as described in the previous sections. Although this works, it is a far from perfect solution.

The high-order model is even more sensitive to any source of oscillations due to lack of explicit diffusion present in both LFA and LEA. The pure hyperbolic nature of the equations forces any non-smoothness (caused by discrete nature of the input data) in the solution to drift in (or out) of the domain.

\section{Comparison results}

In this section we compare the simulation results obtained with the fluid models and the PIC/MC model. As mentioned before, the simulations are performed in nitrogen and neon at STP. For Ne we consider the following externally applied electric fields: $130,170,210,350$ and $460 \mathrm{Td}$, while for $\mathrm{N}_{2}$ we consider electric fields of 350, 460, 590, 770 and $1000 \mathrm{Td}$. We use higher fields for nitrogen because it is a molecular gas, while neon is a noble gas.

Before we proceed to the actual comparisons, we would like to emphasize one more time that the same collisional cross-sections have been used for the particle model as for the generation of the transport data for the fluid models.

\subsection{Basic comparison of planar fronts in $\mathrm{Ne}$}

In figure 4 the velocities of planar fronts as a function of the external reduced electric field for $\mathrm{Ne}$ and $\mathrm{N}_{2}$ are shown. These velocities are calculated by following the time evolution of a certain level $\left(2 \times n^{*}\right.$, see equation (23)) of the electron density at the streamer front. In both gases, the LFA model shows the largest deviation with the PIC/MC results. The front velocity is always too low with this model, with larger deviations at higher fields.

The LEA and high-order model perform about equally good. Note that in neon their predictions are remarkably similar. For lower fields in nitrogen, LEA is almost indistinguishable from PIC/MC, while for higher fields it is about $10 \%$ slower. On the other hand, the high order model slightly overestimates the velocity, and has a better agreement with PIC/ $\mathrm{MC}$ at higher fields.

As expected, the LFA model has the lowest front velocities: with the local field approximation, there is no energy transport. The LEA and high-order model include energy transport, which leads to higher electron energies at the edge of the front, and thus faster growth.

In figure 5 we show the relative difference of the electron density in the streamer channel compared with PIC/MC as a 

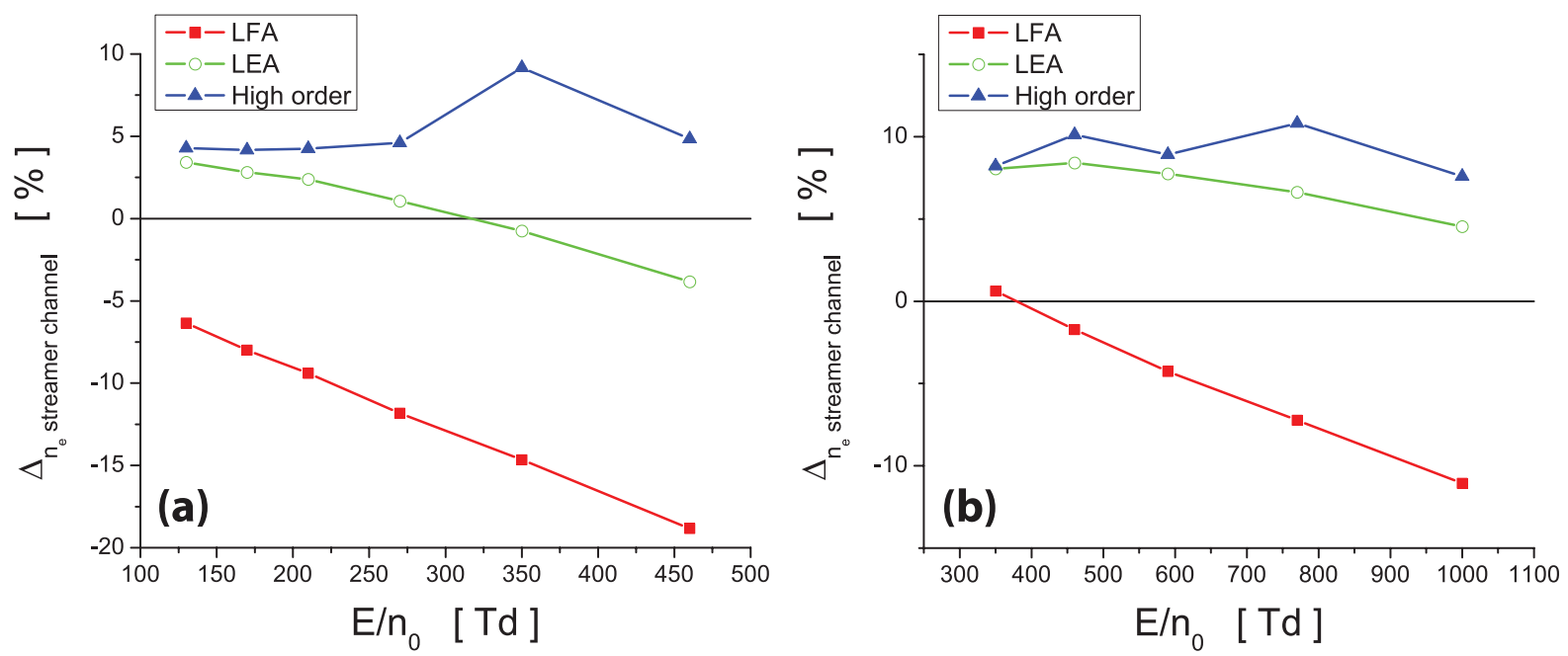

Figure 5. The relative differences of the electron densities in the channel of the negative planar fronts from all fluid models compared with $\mathrm{PIC} / \mathrm{MC}$ as a function of reduced electric field in $\mathrm{Ne}$ (a) and in $\mathrm{N}_{2}$ (b).
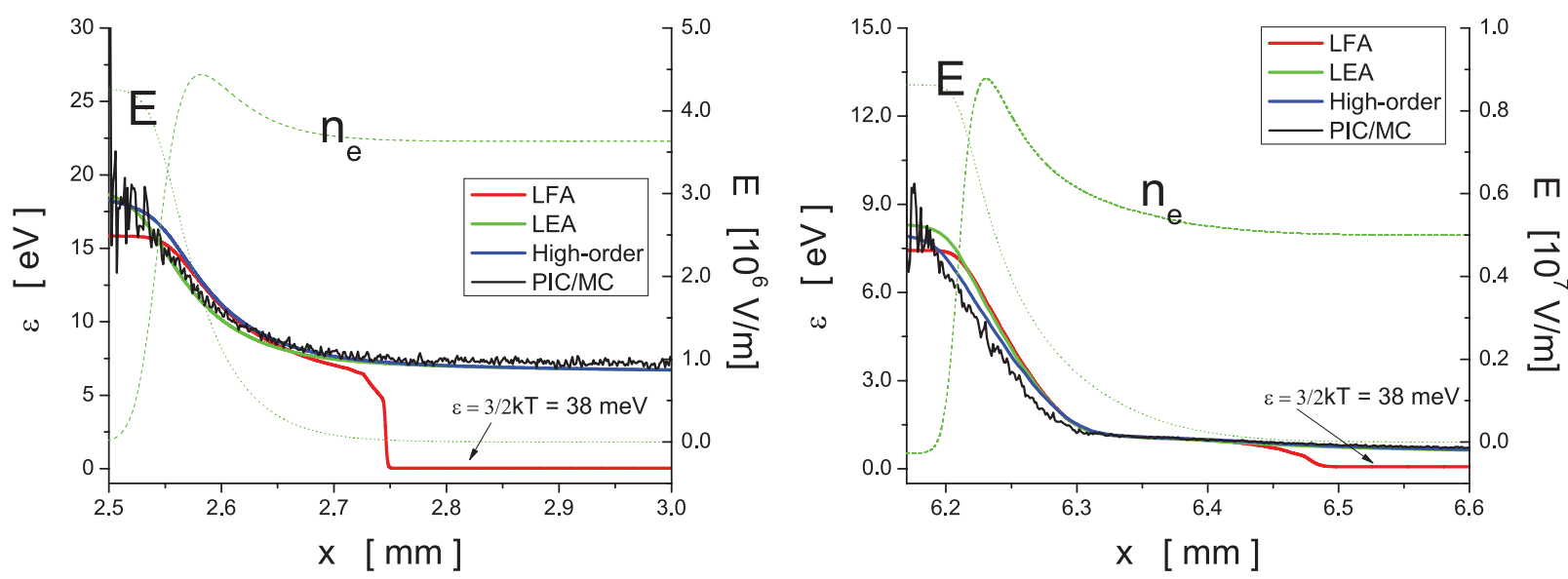

Figure 6. The average electron energy in the four different models. Left: results for neon at $8.5 \mathrm{~ns}$ and $170 \mathrm{Td}$. Right: results for nitrogen at $6.0 \mathrm{~ns}$ and $350 \mathrm{Td}$. Profiles of the electric field and the electron number density from the high order fluid model are also included, to indicate the location of the front. For clarity, the energy profiles are shifted to align with the PIC/MC curve.

function of $E / n_{0}$. In neon, the LFA model shows the largest deviation: it systematically underestimates the electron density by up to $18 \%$ and $13 \%$ for $\mathrm{Ne}$ and $\mathrm{N}_{2}$ respectively. The difference is larger for higher electric fields. The LEA model does slightly better than high-order model, with both showing deviations of up to $5 \%$.

In nitrogen, the LFA model shows the best agreement for lower electric fields. At higher electric fields, it underestimates the electron density in the channel. On the other hand, the LEA and high-order model overestimate the electron density. The LEA model does slightly better, and its results seem to improve for higher fields.

\subsection{Comparison of average electron energies in different models}

In many applications it is important to be able to correctly calculate the space resolved profiles of the average electron energies. The LEA and high order fluid model can calculate such profiles as they contain an equation for the electron energy density, unlike the LFA model. If we assume that in the LFA model the electron energy instantaneously relaxes to the electric field, we can calculate electron energies directly from the field. In figure 6 electron mean energy profiles for the four models (LFA, LEA, high order and PIC/MC) are presented, at $8.5 \mathrm{~ns}$ in $\mathrm{Ne}$ at $170 \mathrm{Td}$. We have also included the profiles of the electric field and the electron density obtained from the high order model to indicate the streamer head position. We have shifted all the profiles to align them with the particle model for comparison.

In figure 6 energy profiles are shown for neon (at $8.5 \mathrm{~ns}, 170$ Td) and nitrogen (at $6.0 \mathrm{~ns}, 350 \mathrm{Td}$ ). The LEA and high-order model give almost the same energy profile in the channel, but near the front the high-order model captures the slope in the mean energy slightly better. Because the high-order model contains balance equations for the momentum and average energy flux, it can give a better description of the front region.

Not surprisingly, the LFA model gives a poor prediction for the mean energy in the channel. The reason is that in this region the electric field is entirely screened in $1 \mathrm{D}$ (when 


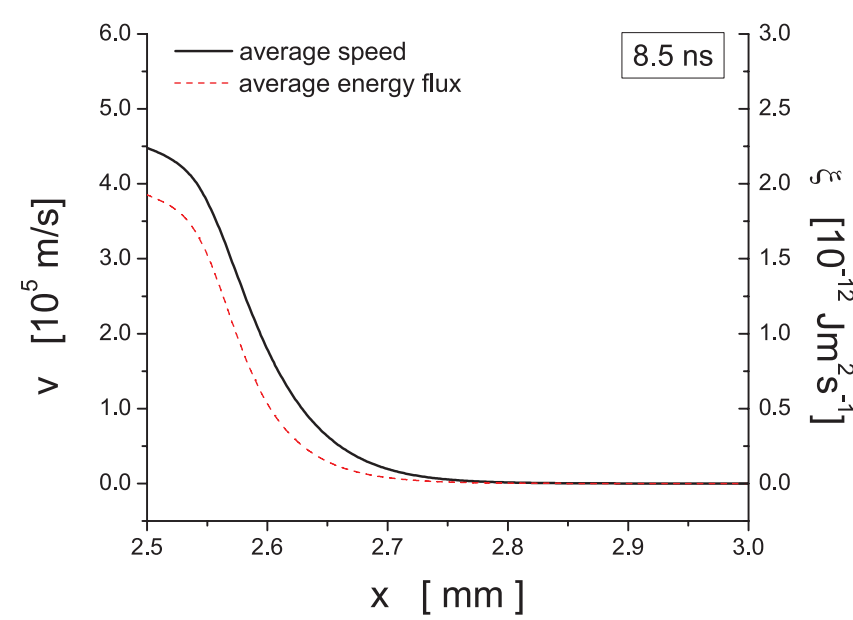

Figure 7. The same instant and plot as in figure 6, but now average electron velocity and average electron energy flux are plotted for $\mathrm{Ne}$ at externally applied electric field of $170 \mathrm{Td}$.

$\partial_{t} E_{0}=0$ ), and therefore LFA predicts a (thermal) mean energy of $0.038 \mathrm{eV}$. What is surprising however, is that the LFA and LEA model predict a similar slope near the front.

The particle model suffers from a lack of electrons where the density goes to zero, and stochastic noise is present. In figure 6 , the particle model predicts a mean electron energy of around $7 \mathrm{eV}$ and $1 \mathrm{eV}$ for $\mathrm{Ne}$ and $\mathrm{N}_{2}$, respectively.

\subsection{Average speed and average energy flux}

In this section we illustrate the features of streamers that are captured by the high order fluid model, namely the average electron speed and average electron energy flux. The average electron speed can be properly described by models that contain a momentum balance equation (i.e. no drift-diffusion approximation) $[13,14,16,19]$. In figure 7 we show the average electron speed and average electron energy flux for $\mathrm{Ne}$. In the region ahead of the front, where there is a constant electric field, the average electron speed have a characteristic slope, a non-local effect. In contrast to this region, in the streamer channel average electron speed is essentially zero. This follows from the much faster electron momentum relaxation than electron energy relaxation. As illustrated by Dujko et al [1] for electrons in $\mathrm{N}_{2}$, the collision frequency for momentum relaxation is almost five orders of magnitude higher than the collision frequency for energy transfer. The average electron energy flux is often explicitly neglected [13, 14, 72]. The importance of the average electron energy flux balance equation was recently highlighted by Becker and Loffhagen [16] and by Markosyan et al [19].

\section{Discussion}

In this paper we have compared three fluid models to Monte Carlo simulations, using input data based on the same cross sections. We have investigated how well the fluid models can capture the physics of an ionization wave in 1D. Our focus was on the physical predictions of the models, not on practical aspects such as ease of implementation, availability of transport data, numerical stability or computational cost. Therefore, our results should not be interpreted as definite advice on which model to use.

Since fluid models of different order have different mathematical morphologies and are non-linearly coupled to the Poisson equation, the sensitivity of results to the used numerical scheme, grid size, time step, boundary condition and spatial dimension can differ. For example, the presence of adaptive (or static) grid refinement will produce more and more uncertainties. In the case of adaptive grid refinement one should study the influence of the refinement criterion on the simulation results [73]. The choice of mesh adaptation can be also an issue $[74,75]$. In the higher dimensions the uncertainties associated with (complex) geometries and roughnesses of the interfaces become very critical. It is very hard to predict how stable certain model will be under these more realistic conditions.

An additional source of uncertainty are the transport coefficients. In earlier studies, it was observed that small differences in cross-sections can produce large differences in plasma parameters $[76,77]$. The sensitivity of the models to transport coefficients should be investigated.

The complexity of models depends on the number of the moments of the Boltzmann equation that are used. The more physics we put in a model, the more complex it becomes: there are more equations to be solved, more initial and boundary conditions to be specified and more input data is required. With the complexity of the system the associated uncertainties also grow. Therefore, it is up to the modeler to decide for given type of discharge and under given conditions (geometries, parameter range, etc) what type of model to consider.

The classical fluid model is equally valid for both the electrons and ions. If ions are going to be included, then the most important ion species must be identified and transport data for these species must be either collected from the literature, or should be calculated by solving Boltzmann's equation or by a Monte Carlo simulation technique. In particular, the ion dynamics play an important role in the propagation of negative planar ionization fronts in light gases as well as for socalled long streamers.

The local energy approximation model for is invalid for ions. The fluid equations in this model are derived assuming the 'classical theory' of charged particle transport which is based on the two-term approximation for solving the Boltzmann equation [78, 79]. However, the two term approximation is never valid for ions as even in elastic collisions there is a large fractional energy exchange between ions and neutral particles. As a consequence, the ion velocity distribution is generally significantly distorted from spherical symmetry in velocity space [80, 81]. The local energy approximation model has been used many times in the past for electrons only while for ions usually the classical fluid model was assumed [13, 14]. Alternatively, one may employ the Wannier relation to relate the ion temperature and temperature of the background gas [82] in association with the Einstein relation for evaluation of the diffusion coefficient [83].

The high-order fluid model is, however, equally valid for ions and electrons. In principle, the RHS of the balance 
equation for ions can be easily modified to account for all relevant collision processes. However, it should be noted that due to strong anisotropy of the ion distribution function in velocity space, the temperature tensor cannot be easily modified to a scalar property. Strictly speaking, two balance equations are required for momentum and two equations for the balance of the energy flux. This in turn will generate new unknowns in the fluid equations and new closure assumptions will be needed to close the system of equations. In addition, collision frequencies for momentum and energy transfers in collisions between ions and neutral particles are required as input. However, for practical purposes model collision operators can be used to derive analytical expressions for the components of temperature tensor as discussed in $[84,85]$.

An integral part of plasma simulations should be verification and validation (V and V) [86, 87]. Indeed, for just this purpose, there has recently been an explicit call for ensuring the fidelity of future simulation tools [22, 88, 89]. In the field of radio frequency discharges some pioneering work has been done by Surendra [90]. Turner et al [91] have developed benchmark solutions for capacitive discharges and showed that a number of independently developed particle-in-cell simulations can reproduce the benchmark solutions. In particular, the swarm literature contains many models suitable for benchmarking plasma models in the free-diffusion limit [23, $52,54,77]$.

Therefore, we believe that uncertainty quantification together with V\&V should be the next step in the modelling of the low-pressure plasmas. These techniques are successfully adopted by related fields such as computational fluid dynamics, computational finance, climate modeling, astrophysics etc [92-97].

\section{Conclusions}

We have compared the performance of three plasma fluid models: a first order model based on the local field approximation (LFA), a second order model based on the local energy approximation (LEA) and a high order model. The test problems we considered were 1D ionization waves in nitrogen and neon, in a wide range of electric fields. As a reference model, we have used a PIC/MC code.

The classical LFA model is the simplest model considered. Despite the simplifying assumptions present in the model and the strong recommendations by Grubert et al [98] to use LEA instead of LFA, we find that using the LFA model gives reasonably good results. Of course, it can not calculate the electron energy, but if one is interested in general characteristics like velocity, ionization level or general shape of the discharge, this model can be the first choice.

Compared to the LFA model, the LEA and high-order model gave better predictions for the discharge velocity. Whereas the LFA model underestimates the ionization density in the channel, these models overestimate this density. Both the LEA and high-order model give good predictions for the energy profile in the channel, but the high order model gives a better description of the energy slope in the discharge front.

\section{Acknowledgments}

AHM and SD acknowledge support from STW-project 10751, part of the Netherlands's Organization for Scientific Research (NWO). SD was also supported by MNRS Projects ON171037 and III41011. It is a pleasure to acknowledge helpful discussions with J van Dijk, A Bourdon, S Pancheshnyi, J A S Witteveen, C Hirsch and $\mathrm{H}$ J Lee. Authors are grateful to M J Kushner for valuable comments and suggestions.

\section{References}

[1] Dujko S, Markosyan A H, White R D and Ebert U 2013 High-order fluid model for streamer discharges: I. Derivation of model and transport data J. Phys. D 46475202

[2] Pasko V 2006 Theoretical modeling of sprites and jets Sprites, Elves and Intense Lightning Discharges (NATO Science Series II: Mathematics, Physics and Chemistry vol 225), ed M Füllekrug et al (Berlin: Springer) pp 253-311

[3] Ebert U and Sentman D D 2008 Streamers, sprites, leaders, lightning: from micro- to macroscales J. Phys. D 41230301

[4] Starikovskiy A and Aleksandrov N 2013 Plasma-assisted ignition and combustion Prog. Energy Combust. Sci. 39 61-110

[5] Kong M G, Kroesen G, Morfill G, Nosenko T, Shimizu T, van Dijk J and Zimmermann J L 2009 Plasma medicine: an introductory review New J. Phys. 11115012

[6] van Heesch E J M, Winands G J J and Pemen A J M 2008 Evaluation of pulsed streamer corona experiments to determine the O* radical yield J. Phys. D 41234015

[7] Li C, Brok W J M, Ebert U and van der Mullen J J A M 2007 Deviations from the local field approximation in negative streamer heads J. Appl. Phys. 101123305

[8] Chanrion O and Neubert T 2008 A PIC-MCC code for simulation of streamer propagation in air J. Comput. Phys. 2277222

[9] Fierro A S, Dickens J C and Neuber A A 2014 3-d simulation of low-temperature plasma development under pulsed conditions IEEE Trans. Plasma Sci. 42 2864-5

[10] Kolobov V I and Arslanbekov R R 2012 Towards adaptive kinetic-fluid simulations of weakly ionized plasmas $J$. Comput. Phys. 231839

[11] Arslanbekov R R, Kolobov V I and Frolova A A 2013 Kinetic solvers with adaptive mesh in phase space Phys. Rev. E 88063301

[12] Schunk R W 1977 Mathematical structure of transport equations for multispecies flows Rev. Geophys. 15 429-45

[13] Eichwald O, Ducasse O, Merbahi N, Yousfi M and Dubois D 2006 Effect of order fluid models on flue gas streamer dynamics J. Phys. D 3999

[14] Kanzari Z, Yousfi M and Hamani A 1998 Modeling and basic data for streamer dynamics in $\mathrm{N} 2$ and $\mathrm{O} 2$ discharges J. Appl. Phys. 844161

[15] Gogolides E and Sawin H H 1992 Continuum modeling of radio-frequency glow discharges. I. Theory and results for electropositive and electronegative gases J. Appl. Phys. 723971

[16] Becker M M and Loffhagen D 2013 Derivation of moment equations for the theoretical description of electrons in nonthermal plasmas Adv. Pure Math. 3 343-52

[17] Kushner M J 2004 Modeling of microdischarge devices: pyramidal structures J. Appl. Phys. 95846 
[18] Li C, Ebert U and Hundsdorfer W 2010 Spatially hybrid computations for streamer discharges with generic features of pulled fronts: I. Planar fronts J. Comput. Phys. 229200

[19] Markosyan A H, Dujko S and Ebert U 2013 High-order fluid model for streamer discharges: II. Numerical solution and investigation of planar fronts J. Phys. D 46475203

[20] Ebert U, van Saarloos W and Caroli C 1997 Propagation and structure of planar streamer fronts Phys. Rev. E 55 1530-49

[21] Ebert U and van Saarloos W 2000 Front propagation into unstable states: universal algebraic convergence towards uniformly translating pulled fronts Physica D 146 1-99

[22] Turner M 2013 Verification and validation in low temperature plasma physics APS Meeting Abstracts $\mathbf{1}$

[23] Dujko S, White R D, Petrović Z L and Robson R E 2010 Benchmark calculations of nonconservative chargedparticle swarms in dc electric and magnetic fields crossed at arbitrary angles Phys. Rev. E 81046403

[24] Winkler R, Loffhagen D and Sigeneger F 2002 Temporal and spatial relaxation of electrons in low temperature plasmas Appl. Surf. Sci. 192 50-71

[25] Robson R E and Ness K F 1986 Velocity distribution function and transport coefficients of electron swarms in gases: spherical-harmonics decomposition of Boltzmann's equation Phys. Rev. A 332068

[26] Ness K F and Robson R E 1986 Velocity distribution function and transport coefficients of electron swarms in gases. II. Moment equations and applications Phys. Rev. A 342185

[27] Dhali S K and Williams P F 1987 Two-dimensional studies of streamers in gases J. Appl. Phys. 624696

[28] Vitello P A, Penetrante B M and Bardsley J N 1994 Simulation of negative-streamer dynamics in nitrogen Phys. Rev. E 495574

[29] Kulikovsky A A 1994 The structure of streamers in $\mathrm{N}_{2}$. II. Two-dimensional simulation J. Phys. D 272564

[30] Kulikovsky A A 1995 Two-dimensional simulation of the positive streamer in $\mathrm{N}_{2}$ between parallel-plate electrodes $J$. Phys. D 282483

[31] Babaeva N Y and Naidis G V 1996 Two-dimensional modelling of positive streamer dynamics in non-uniform electric fields in air J. Phys. D 292423

[32] Celestin S, Bonaventura Z, Zeghondy B, Bourdon A and Ségur P 2009 The use of the ghost fluid method for Poisson's equation to simulate streamer propagation in point-to-plane and point-to-point geometries J. Phys. D 42065203

[33] Pancheshnyi S, Ségur P, Capeillére J and Bourdon A 2008 Numerical simulation of filamentary discharges with parallel adaptive mesh refinement J. Comput. Phys. 2276574

[34] Eichwald O, Bensaad H, Ducasse O and Yousfi M 2012 Effects of numerical and physical anisotropic diffusion on branching phenomena of negative-streamer dynamics J. Phys. D 45385203

[35] Naidis G V 1997 Effects of nonlocality on the dynamics of streamers in positive corona discharges Tech. Phys. Lett. 23493

[36] Aleksandrov N L and Kochetov I V 1996 Electron rate coefficients in gases under non-uniform field and electron density conditions J. Phys. D 291476

[37] Abbas I and Bayle P 1980 A critical analysis of ionising wave propagation mechanisms in breakdown J. Phys. D 131055

[38] Abbas I and Bayle P 1981 Non-equilibrium between electrons and field in a gas breakdown ionising wave. I. Macroscopic model J. Phys. D 14649

[39] Bayle P and Cornebois B 1985 Propagation of ionizing electron shock waves in electrical breakdown Phys. Rev. A 311046
[40] Hagelaar G J M and Pitchford L C 2005 Solving the Boltzmann equation to obtain electron transport coefficients and rate coefficients for fluid models Plasma Sources Sci. Technol. 14722

[41] Boeuf J P and Pitchford L C 1995 Two-dimensional model of a capacitively coupled rf discharge and comparisons with experiments in the gaseous electronics conference reference reactor Phys. Rev. E $\mathbf{5 1} 1376$

[42] Davoudabadi M, Shrimpton J S and Mashayek F 2009 On accuracy and performance of high-order finite volume methods in local mean energy model of non-thermal plasmas J. Comput. Phys. 2282468

[43] van Dijk J, Peerenboom K, Jimenez M, Mihailova D and van der Mullen J 2009 The plasma modelling toolkit Plasimo J. Phys. D 42194012

[44] Kim H C, Iza F, Yang S S, Radmilovic-Radjenovic M and Lee J K 2005 Particle and fluid simulations of lowtemperature plasma discharges: benchmarks and kinetic effects J. Phys. D 38 R83

[45] Hamaguchi S 1999 Modeling and simulation methods for plasma processing IBM J. Res. Dev. 43 199-215

[46] Raizer Y P, Shneider M N and Yatsenko N A 1995 RadioFrequency Capacitive Discharges (London: Taylor and Francis)

[47] Bhoj A N and Kushner M J 2004 Avalanche process in an idealized lamp: II. Modelling of breakdown in $\mathrm{Ar} / \mathrm{Xe}$ electric discharges J. Phys. D 372510

[48] Wen-Xia S, Qing-Jun P, Qing Y, Tao Y and Jian S 2013 Local electron mean energy profile of positive primary streamer discharge with pin-plate electrodes in oxygen-nitrogen mixtures Chin. Phys. B 22015203

[49] Guo J-M and Wu C-H J 1993 Two-dimensional nonequilibrium fluid models for streamers IEEE Trans. Plasma Sci. 21684

[50] Hagelaar G J M and Kroesen G M W 2000 Speeding up fluid models for gas discharges by implicit treatment of the electron energy source term J. Comput. Phys. 159 1-12

[51] Becker M M and Loffhagen D 2013 Enhanced reliability of drift-diffusion approximation for electrons in fluid models for nonthermal plasmas AIP Adv. 3012108

[52] White R D, Robson R E, Dujko S, Nicoletopoulos P and Li B 2009 Recent advances in the application of boltzmann equation and fluid equation methods to charged particle transport in non-equilibrium plasmas J. Phys. D 42194001

[53] Robson R E 1986 Physics of reacting particle swarms in gases J. Chem. Phys. 854486

[54] Robson R E, White R D and Petrović Z L 2005 Colloquium: physically based fluid modeling of collisionally dominated low-temperature plasmas Rev. Mod. Phys. 771303

[55] Dujko S, Ebert U, White R D and Petrović Z L 2011 Boltzmann equation analysis of electron transport in a $\mathrm{N}_{2}-\mathrm{O}_{2}$ streamer discharge Japan. J. Appl. Phys. $5008 \mathrm{JC} 01$

[56] Li C, Ebert U and Hundsdorfer W 2012 Spatially hybrid computations for streamer discharges : II. Fully 3D simulations J. Comput. Phys. 2311020

[57] Hayashi M 2000 private communication

[58] Ebert U and van Saarloos W 2000 Breakdown of the standard perturbation theory and moving boundary approximation for pulled fronts Phys. Rep. 337 139-56

[59] Koren B 1993 A robust upwind discretization method for advection, diffusion and source terms Notes Numer. Fluid Mech. 45 117-38

[60] Toro E F, Hidalgo A and Dumbser M 2009 FORCE schemes on unstructured meshes I: conservative hyperbolic systems J. Comput. Phys. 228 3368-89

[61] Dumbser M, Hidalgo A, Castro M, Pars C and Toro E F 2010 FORCE schemes on unstructured meshes II: nonconservative hyperbolic systems Comput. Methods Appl. Mech. Eng. 199 625-47 
[62] Sokol Z 1999 Comparison of several numerical schemes applied to advection equations $Q$. J. R. Meteorol. Soc. 125 213-24

[63] Rubio A D, Zalts A and El Hasi C D 2008 Numerical solution of the advection-reaction-diffusion equation at different scales Environ. Modelling Softw. 23 90-5

[64] Bouche D, Bonnaud G and Ramos D 2003 Comparison of numerical schemes for solving the advection equation Appl. Math. Lett. 16 147-54

[65] Woodward P and Colella P 1984 The numerical simulation of two-dimensional fluid flow with strong shocks J. Comput. Phys. 54 115-73

[66] Wilcoxson M H and Manousiouthakis V I 1996 Simulation of a three-moment fluid model of a two-dimensional radio frequency discharge Chem. Eng. Sci. 51 1089-106

[67] Hundsdorfer W and Verwer J G 2003 Numerical Solution of Time-Dependent Advection-Diffusion-Reaction Equations (Springer Series in Computational Mathematics) (Berlin: Springer)

[68] Montijn C, Hundsdorfer W and Ebert U 2006 An adaptive grid refinement strategy for the simulation of negative streamers J. Comput. Phys. 219801

[69] Barnes M S, Cotler T J and Elta M E 1987 Large signal time domain modeling of low pressure rf glow discharges J. Appl. Phys. 61 81-9

[70] Courant R, Friedrichs K and Lewy H 1928 Über die partiellen differenzengleichungen der mathematischen physik Math. Ann. 100 32-74

[71] Hagelaar G J M 2000 Modeling of microdischarges for display technology PhD Thesis Technische Universiteit Eindhoven

[72] Yousfi M, Hennad A, Benhenni M, Eichwald O and Merbahi N 2012 Basic data of ions in He-air mixtures for fluid modeling of low temperature plasma jets $J$. Appl. Phys. 112043301

[73] Trompert R and Verwer J 1993 Analysis of the implicit euler local uniform grid refinement Method SIAM J. Sci. Comput. 14 259-78

[74] Huang W 2001 Variational mesh adaptation: isotropy and equidistribution J. Comput. Phys. 174 903-24

[75] Huang W and Sun W 2003 Variational mesh adaptation II: error estimates and monitor functions $J$. Comput. Phys. 184 619-48

[76] Bose D, Rao M V V S, Govindan T R and Meyyappan M 2003 Uncertainty and sensitivity analysis of gas-phase chemistry in a $\mathrm{CHF}_{3}$ plasma Plasma Sources Sci. Technol. 12225

[77] Lj Petrović Z, Dujko S, Marić D, Malović G, Nikitović Ž, Šašić O, Jovanović J, Stojanović V and RadmilovićRaenović M 2009 Measurement and interpretation of swarm parameters and their application in plasma modelling J. Phys. D: Appl. Phys. 42194002

[78] Allis W P 1967 Electrons, Ions, and Waves: Selected Papers of William Phelps Allis (Cambridge, MA: MIT Press)

[79] Alves L L 2007 Fluid modelling of the positive column of direct-current glow discharges Plasma Sources Sci. Technol. 16557

[80] White R D, Robson R E, Schmidt B and Morrison M A 2003 Is the classical two-term approximation of electron kinetic theory satisfactory for swarms and plasmas? J. Phys. D 363125
[81] White R D, Robson R E and Ness K F 2001 Computation of electron and ion transport properties in gases Comput. Phys. Commun. 142349

[82] Wannier G H 1953 Motion of gaseous ions in strong electric fields Bell Syst. Tech. J. 32 170-254

[83] Sobota A, Manders F, van Veldhuizen E M, van Dijk J and Haverlag M 2010 The role of metastables in the formation of an argon discharge in a two-pin geometry IEEE Trans. Plasma Sci. 382289

[84] Robson R E 1994 Approximate formulas for ion and electron transport coefficients in crossed electric and magnetic fields Aust. J. Phys. 47 279-304

[85] Jovanović J V, Vrhovac S B and Petrović Z L 2002 Momentum transfer theory of ion transport under the influence of resonant charge transfer collisions: the case of argon and neon ions in parent gases Eur. Phys J. D 21335

[86] Roache P J 1998 Verification and Validation in Computational Science and Engineering (Albuquerque, NM: Hermosa)

[87] Oberkampf W L and Roy C J 2010 Verification and Validation in Scientific Computing (Cambridge: Cambridge University Press)

[88] Turner M M and Vukovic M 2012 Overview of verification and validation in low temperature plasma physics APS Meeting Abstracts 13001

[89] Samukawa S et al 2012 The 2012 plasma roadmap J. Phys. D 45253001

[90] Surendra M 1995 Radiofrequency discharge benchmark model comparison Plasma Sources Sci. Technol. 456

[91] Turner M M, Derzsi A, Donkó Z, Eremin D, Kelly S J, Lafleur T and Mussenbrock T 2013 Simulation benchmarks for low-pressure plasmas: Capacitive discharges Phys. Plasmas 20013507

[92] Beven K and Binley A 1992 The future of distributed models: model calibration and uncertainty prediction Hydrol. Process. 6 279-98

[93] Murphy J M, Sexton D M H, Barnett D N, Jones G S, Webb M J, Collins M and Stainforth D A 2004 Quantification of modelling uncertainties in a large ensemble of climate change simulations Nature $430768-72$

[94] de Jong H 2002 Modeling and simulation of genetic regulatory systems: a literature review J. Comput. Biol. 9 67-103

[95] Oberkampf W L, DeLand S M, Rutherford B M, Diegert K V and Alvin K F 2000 Estimation of total uncertainty in modeling and simulation Sandia Report SAND2000-0824, (Albuquerque, NM)

[96] Oberkampf W L, DeLand S M, Rutherford B M, Diegert K V and Alvin K F 2002 Error and uncertainty in modeling and simulation Reliab. Eng. Syst. Saf. 75 333-57

[97] Calder A C, Fryxell B, Plewa T, Rosner R, Dursi L J, Weirs V G, Dupont T, Robey H F, Kane J O and Remington B A 2002 On validating an astrophysical simulation code Astrophys. J. Suppl. Ser. 143201

[98] Grubert G K, Becker M M and Loffhagen D 2009 Why the local-mean-energy approximation should be used in hydrodynamic plasma descriptions instead of the local-field approximation Phys. Rev. E 80036405 